\begin{tabular}{ccc}
\hline International Journal of Engineering \& Technology, 7 (2.32) (2018) $466-469$ \\
SPC & Website: www.sciencepubco.com/index.php/IJET \\
Research paper & Technology \\
\hline
\end{tabular}

\title{
An Expert System to Mitigate Problems in University Using Chat Bot
}

\author{
S.Akhil ${ }^{1}$, V.Sai Krishna ${ }^{2}$, M.Kranthi ${ }^{3}$, A.Nihar ${ }^{4}$, T.S.Mastan Rao ${ }^{5}$ \\ 1,2,3,4,5 Department of CSE, KLEF, Andhra Pradesh, India \\ *Corresponding author E-mail: akhilsuda@gmail.com
}

\begin{abstract}
There has been a rapid increase in the data generated over the past few decades in all domains. As part of our research study we are considering domain of information provided by the university to the public i.e. the aspirants applying to the university as well as the students undergoing the curriculum. We are developing an advanced chat-bot which assists the students to gather information about the university. Since the information which can be provided from the official website is not up to the mark, there is a need for developing another medium for providing the necessary information. Whenever a student requires some assistance of any matter regarding university they can gather information from helpdesk, website or billboards. Sometimes the information may not be accurate and insufficient. So, in order to make the required information accessible to the students we are developing this software where students can interact with the software assistant through text and gain the information that is required by them. The software allows the student to interact with it in text basis and provides almost any information regarding University.
\end{abstract}

Keywords:Expert System, Chat Bot.

\section{Introduction}

Chat-bot is a conversational agent that interacts with substance users using natural language(NL). This applied science which avail humans converse with users in their natural language through a computer interface. The rise of electronic messaging apps, the expansion of the app ecosystem, advance in artificial intelligence and cognitive technologies, a fascination with conversational user interfaces and a wider reach of automation are all driving the Chatbot . It is a information processing system course of study conception to simulate an intelligent conversation with a human user in a auditory or textual methods. Most of the chat bots are designed for engaging in small talks and their personalities are created by the computer programmer. The good introduction in designing chat bots using the current state of the nontextual matter which is mainly used rules written in AIML(artificial intelligent markup language).

\section{Various Existing Chat Bot}

MITSUKU: This chat bot is one of the best Ai chat bots. Evidently it is the current winner of LOEBNER PRIZE. It is a bot made to chat about anything, which is one of the main reasons that make it so human like - contrary to other chat bots that are made for a specific task.[2]

Casper: CASPER is a messenger bot designed to be your one and only weather expert. It sends alerts up to twice a day with user consent and is intelligent enough to a questions like "should I take an umbrella today?"[7]
RIGHT CLICK: Right Click is a startup that introduced an A.I.powered Chabot that creates websites. It asks general questions during the conversation like "What industry you belong to?" and "Why do you want to make a website?" and creates customized templates as per the given answers. Hira Saeed tried to divert it from its job by asking it about love, but what a smart player it is! By replying to each of her queries, it tried to bring her back to the actual job of website creation. The process was short but keeps you hooked.

The Optimizer: This is by far the largest category of functional bots. In a way, all other categories are spin-offs of this one. These bots take on some concrete challenge and try solving it better than existing apps or websites. For example, streaming music in your living room by saying "Alexa, play my library" is more convenient than clicking a couple of buttons on your mobile device to achieve the same result. We are seeing multiple brands toying with the messaging canvas for shopping, traveling and many other use-cases. These bots attempt to disrupt by reducing friction versus more traditional ways of 'doing things.' [4]

Alice: Alice is a natural speech communication processing chatterbot a program that engages in a conversation with a human being by applying some heuristicall pattern matching principle to the human's input , and in its online form it also relies on a hidden third person. It was inspired by Joseph Weizenbaum's classical ELIZA program. It is one of the strongest programs of its type and has win the Loebner Prize, awarded to accomplished humanoid. However, the program is unable to pass the Turing trial, as even the casual user will often unmasking its mechanistic aspects in short conversations.[1] 


\section{Literature Review:}

This is a Chatbot made by Steve Worswick. Obviously it is the present champ of LOEBNER PRIZE. This is made to visit about anything, The reason that make it so human like - as opposed to other talk bots that are made for a particular errand. This Chatbot has an unpredictable calculation where it separates each word and deduces it importance and answer appropriately [2].

CSIEC (Computer Simulation in Educational Communication) framework with recently built up numerous capacities for English guideline still spotlights on providing a virtual visiting accomplice (Chatbot), which can talk in English with the English students anytime anywhere. It produces informative reaction as indicated by the client input, the exchange setting, the client's and its own identity learning, sound judgment learning, and inference knowledge. [3] This is a startup that presented an A.I.- controlled Chatbot that makes sites. It makes general inquiries amid the discussion like "What industry you have a place with?" and "For what reason would you like to make a site?" and makes modified layouts according to the given answers. Hira Saeed endeavored to redirect it from its activity by getting some information about affection, however what a keen player it is! By answering to each of her inquiries, it attempted to take her back to the genuine activity of site creation. The procedure was short yet keeps you snared.[9]

PONCHO is a messenger bot intended to be your unrivaled climate master. It sends alarms up to twice per day with client assent and is sufficiently clever to answer questions like "would it be a good idea for me to take an umbrella today?".[6]

This Chatbot presents a PC execution that uses cross-reference equivocalness in articulations for basic conversational jokes. The approach depends on the SSTH. Utilizing a basic content portrayal, it is demonstrated that cross-reference ambiguities dependably fulfil1 the SSTH necessity for content cover. Because of the low execution of the anaphora determination calculation and the information inadequacy in ConceptNet the application performs reasonably, yet it does not provide future prospects in creating conversational diversion.[11]

Insomno bot is for night owls. As the title recommends, it is for all individuals out there who have inconvenience resting. This bot talks to you when you have no one around and gives you astonishing answers so that you won't get bored. It's not something that will offer assistance you check stars when you can't rest or offer assistance you with perusing recommendations, but this bot talks to you about anything.[7]

Disney: Solving Crimes with Fictional Characters in which Disney invited fans of that movie to solve crimes with Lieutenant Judy Hopps, the tenacious, long-eared protagonist of the movie. Childre$\mathrm{n}$ could help Lt. Hopps investigate mysteries like those in the movie by interacting with the bot, which explored avenues of inquiry based on user input. Users can make suggestions for Lt. Hopps' investigations, to which the chatbot would respond. [12]

\section{Methodology}

most of the source code is taken up by a word reference called reflections and a list of records called psychobabble. This bot is on a very basic level a design coordinating program. There is not much more to it than that. reflections maps first-person pronouns to second-person pronouns and vice-versa. It is utilized to "reflect" a explanation back against the client.

psychobabble is formed from a listing of lists wherever the primary part may be a regular expression that matches the user's statements and also the second part may be a list of potential responses. several of the potential responses contain placeholders which will be stuffed in with fragments to echo the user's statements.

Main is the entry point of the program. Let's take a closer look at it. def main():

print ("Hello")

while True:

statement $=$ raw_input $(">$ ")

print analyze(statement)

if statement $==$ "quit":

break

First, we tend to print the initial prompt, then we tend to enter a loop of asking the user for input and spending what the user says to the analyze operate to urge the therapist's response. If at any purpose the user varieties "quit", we tend to run off of the loop and also the program exits.

Let's see what's going on in analyze.

def analyze(statement):

for pattern, responses in psychobabble:

match = re.match(pattern, statement.rstrip(".!"))

if match:

response $=$ random. choice $($ responses $)$

return

response.format $(*[\operatorname{reflect}(\mathrm{g})$ for $\mathrm{g}$ in match.groups ()$])$

We repeat through the regular expressions within the jargon array, attempting to match each with the user's statement, from that we've got stripped the ultimate punctuation. If we discover a match, we select a response guide indiscriminately from the list of potential responses related to the matching pattern. Then we have a tendency to interpolate the match terms from the regular expression into the response string, vocation the replicate operate on every match cluster 1 st.

There is one syntactic peculiarity to note here. When we utilize the rundown understanding to produce a rundown of reflected match gatherings, we detonate the rundown with the reference mark (*) character before passing it to the string's configuration strategy. Organization expects a progression of positional contentions relating to the quantity of arrangement placeholders $-\{0\},\{1\}$, and so on - in the string. A rundown or a tuple can be detonated into positional contentions utilizing a solitary reference mark. Twofold marks $(* *)$ can be utilized to detonate lexicons into catchphrase contentions.

Now comes the reflect function. There is nothing excessively confounded going ahead in it. In the first place, we put forth the expression lowercase, at that point we tokenize it by part on whitespace characters. We emphasize through the rundown of tokens and, if the token exists in our appearance word reference, we supplant it with the incentive from the lexicon. So "I" moves toward becoming "you", "your" turns into "my", and so on.

As should be obvious, this is a basic program. The main genuine knowledge in it is associated with the making of appropriately dubious reaction formats. Have a go at fiddling with the psychobabble rundown to expand it's conversational range and give it an alternate tone. 


\section{Architecture}

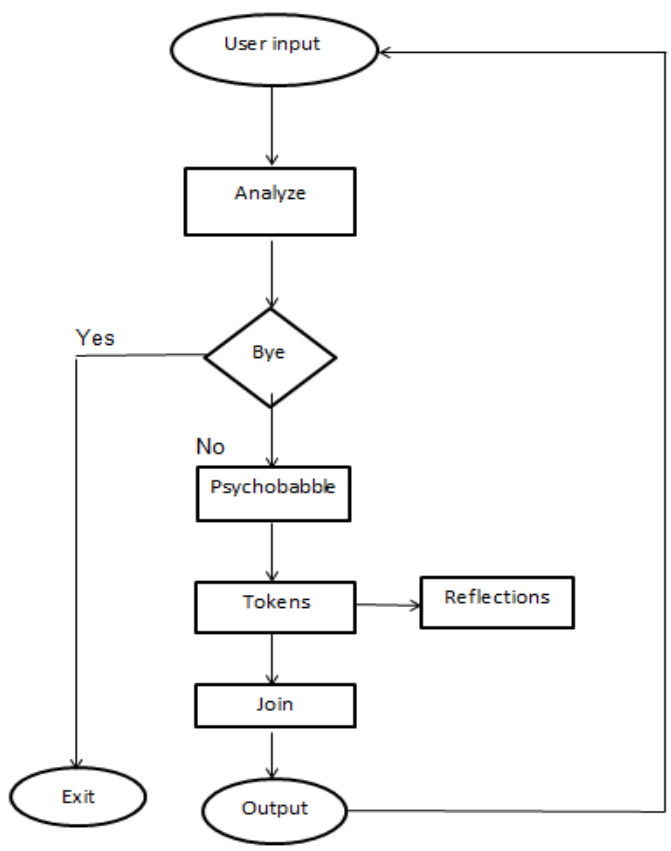

In our program first user will enter some text as input then the text will be analyzed. After analyzing the text if the text entered is "Bye" then the program will quit otherwise, it will try to match the sentence entered with the sentence in the psychobabble function. If entered text is found then it will choose one reply randomly from the set of options available. Then it will tokenize the text sentence and try to match each token word with the words in the reflections and if the word matches the word in reflection then it will replace the word with the one next to it in reflection. Now it will join all the tokenized words and display as output to the user and waits for the user input. This is how our program works.

\section{Algorithm:}

1. Start

2. Wait for the user input.

3. 3.If the text entered is "Bye" then go to step 9.

4. When user inputs some text or sentence first cross reference it with the sentences that are in psychobabble program.

5. If the user sentence matches to the sentence in psychobabble then select a random output from the set of outputs.

6. When the sentence match in the psychobabble other than the words matched to the user inputted sentence for some inputs the remaining words will be copied and pasted next to the randomly selected output.

7. Now this sentence will be tokenized then each and every word will be cross referenced with words that are present in the reflections function. If the word matches then the word will be replaced with word next to it in reflections and then all the words will be joined

8. Now the output is shown to user and return to step 2.

9. Display "Thank you for talking with me"

10. Stop.

We repeat through the algorithm within the jargon array, attempting to match each with the user's statement, from that we've got stripped the ultimate punctuation. If we discover a match, we select a response guide indiscriminately from the list of potential responses related to the matching pattern. Then we have a tendency to interpolate the match terms from the regular expression into the response string, vocation the replicate operate on every match cluster 1 st.
This algorithm describes explicitly all the actions that will be processing in the program.

\section{Results}

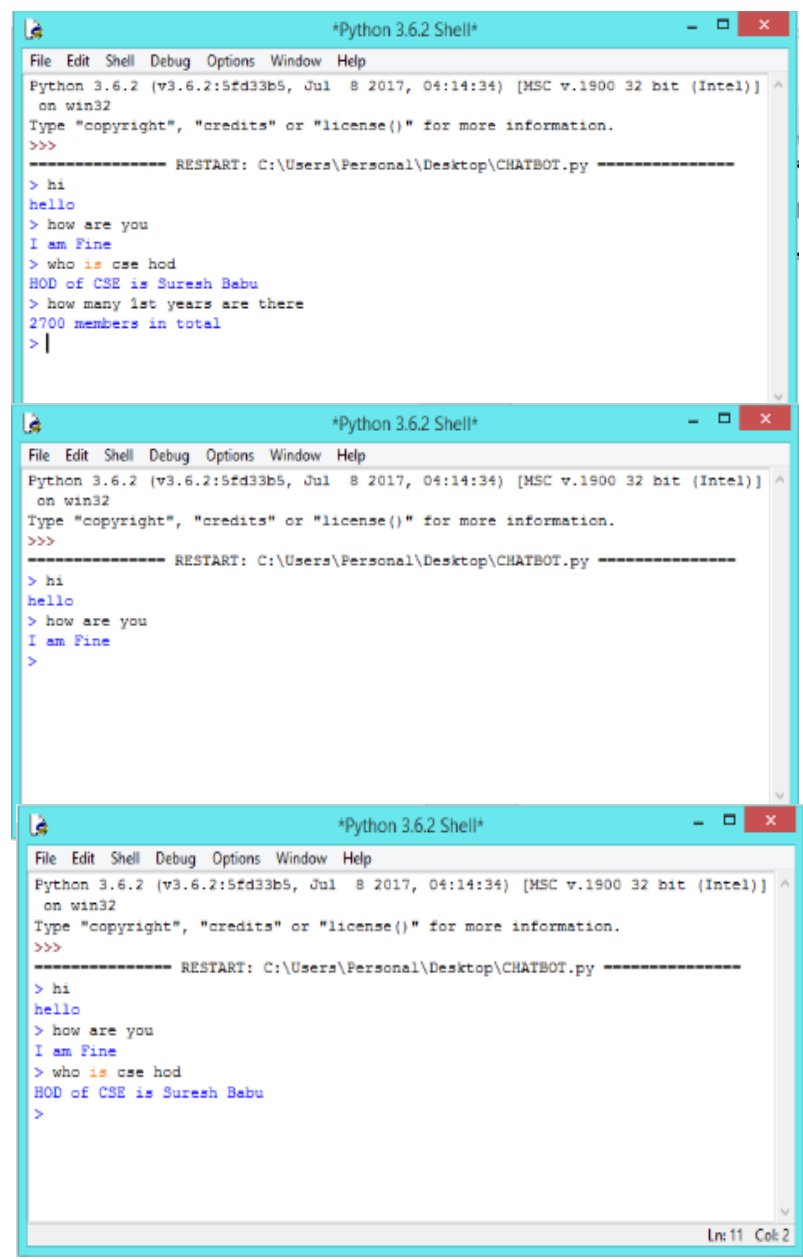

\section{References}

[1] ALICE. Http://www.alicebot.org/.

[2] MITSUKI.Http://www.mitsuki.org/.

[3] Jiyou jia , 2009, A computer assisted English learning chatbot based on textual knowledge and reasoning

[4] Buckingham Shum, S., MacLean, A., Bellotti, V. M. E., and Hammond, N. V. 1997. Graphical argumentation and design cogni-tion. Human-Computer Interaction, 12(3), 1997, 267- 300.

[5] Prager, J. M., Chu-Carroll, J., and Czuba, K.W.. 2004. Ques-tion answering using constraint satisfaction. Proceedings of the 42 nd Meeting of the Association for Computational Linguistics (ACL'04), 2004.

[6] Xu, J., Licuanan, A., Weischedel, R. 2003. TREC 2003 QA at BBN Answering Definitional Questions. Proceedings of TREC 2003.

[7] INSOMNIA. https://www.psfk.com/2016/10/insomnia-bot-helpsyou-sleep.html

[8] MITSUKU. https://en.wikipedia.org/wiki/Mitsuku.

[9] Moldovan, D., Harabagiu, S., Pasca, M., Mihalcea, R., Girju, R., Goodrum, R., and Rus, V. 2000. The structure and performance of an open-domain question answering system. Proceedings of ACL2000.

[10] Xu, J., Licuanan, A., Weischedel, R. 2003. TREC 2003 QA at BBN: Answering Definitional Questions. Proceedings of TREC 2003.

[11] Hans Wim Tinholt, Anton Nijholt. Computational Humour: Utilizing Cross-Reference Ambiguity for Conversational Jokes, 2007. 
[12] Poggi, I., and Pelachaud, C. 2000. Emotional meaning and expression in performative faces.

[13] A.Paiva ed.: Affective interactions: Towards a new generation of computer interfaces. Springer Verlag, Berlin, Heidelberg, New York

[14] Kerly, P. Hall, S. Bull, Bringing chatbots into education: towards natural language negotiation of open learner models, Knowledge Based Systems 20 (2) (2007) 177-185

[15] W. Chen, J. Jia, D. Xiang, The application of CSIEC in Eng-lish instruction in high school: design based research, China Educational Technology 20 (2) (2008) 109-114.

[16] Ministry of Education China, Annual Educational Statistics 2006, People's Education Press, Beijing, 2006.

[17] L. Fryer, R. Carpenter, Emerging technologies bots as lan-guage learning tools, Language Learning and Technology 10 (3) (2006) $8-14$.

[18] P. Kerfoot et al., A multi-institutional randomized controlled trial of webbased teaching to medical students, Academic Medicine 81 (3) (2006) 224-230.

[19] Plutchik, R. 1980. A general psychoevolutionary theory of emotion. Plutchik, R. and Kellerman, H. (eds.): Emotion theory, research, and experience. 3-33 pp. Academic Press.

[20] Tatai, G., Csord·s, A., Kiss, £., Laufer, L., and SzalÛ, A. 2003. The chatbot who loved me. to appear in the proceedings of the ECA workshop of AAMAS 2003.

[21] Mulder, M. P., and Nijholt, A. 2002. Humour research: State of the art. CTIT Technical Report series 02-34. 